\title{
HOPF FORMULA AND MULTITIME HAMILTON-JACOBI EQUATIONS
}

\author{
P. L. LIONS AND J-C. ROCHET
}

\begin{abstract}
Problems arising in mathematical economics lead to the study of multitime Hamilton-Jacobi equations. Using commutation properties of the semigroups for the standard equation, we propose a generalization of the Hopf formula that gives explicit solutions of these equations.
\end{abstract}

I. Hopf formula. We consider the following Cauchy problem for the HamiltonJacobi equation:

$$
\begin{array}{ll}
\partial_{u} / \partial t+H(D u)=0 & \text { in } \mathbf{R}^{N} \times[0, T], \\
u(x, 0)=u_{0}(x) & \text { in } \mathbf{R}^{N} .
\end{array}
$$

M. G. Crandall and P. L. Lions [2, 3] introduced the notion of viscosity solution of (1): In particular, they proved the existence and uniqueness of a viscosity solution of (1) in $\operatorname{BUC}\left(\mathbf{R}^{N} \times[0, T]\right)$ when $H$ is continuous on $\mathbf{R}^{N}$ and $u_{0}$ belongs to $\operatorname{BUC}\left(\mathbf{R}^{N}\right)$, the set of functions bounded and uniformly continuous on $\mathbf{R}^{N}$. This solution is denoted by $\left(S_{H}(t) u_{0}\right)(x)$. Of course, the family $\left(S_{H}(t)\right)_{t \geqslant 0}$ defines a strongly continuous semigroup on $\mathrm{BUC}\left(\mathbf{R}^{N}\right)$.

When $u_{0}$ is in $\operatorname{BUC}\left(\mathbf{R}^{N}\right)$ and $H$ is convex and coercive (i.e., $\lim _{|p| \rightarrow+\infty} H(p) /|p|$ $=+\infty)$, this viscosity solution is given explicitly by the Oleinik-Lax formula (cf. [7])

$$
u(x, t)=\operatorname{Inf}_{y \in \mathbf{R}^{N}} \operatorname{Sup}_{z \in \mathbf{R}^{N}}\left\{u_{0}(y)+(z, x-y)-t H(z)\right\} .
$$

A proof of this fact may be found in Lions [8]. This can also be written

$$
u(x, t)=\operatorname{Inf}_{y \in \mathbf{R}^{N}}\left\{u_{0}(y)+t H^{*}\left(\frac{x-y}{t}\right)\right\}
$$

where $H^{*}$ is the Fenchel conjugate of $H$ :

$$
H^{*}(p)=\operatorname{Sup}_{z \in \mathbf{R}^{N}}\{(p, z)-H(z)\} .
$$

On the other hand, when $u_{0}$ is convex and $H$ is continuous, Hopf [5] proposed the following formula (dual of (2)):

$$
v(x, t)=\operatorname{Sup}_{z \in \mathbf{R}^{N}} \operatorname{Inf}_{y \in \mathbf{R}^{N}}\left\{u_{0}(y)+(z, x-y)-t H(z)\right\} .
$$

Received by the editors September 17, 1984.

1980 Mathematics Subject Classification. Primary 35L60, 35L45, 35F20, 49 C05.

(C) 1986 American Mathematical Society $0002-9939 / 86 \$ 1.00+\$ .25$ per page 
This can also be written

$$
v(x, t)=\operatorname{Sup}_{z \in \mathbf{R}^{N}}\left\{(x, z)-u_{0}^{*}(z)-t H(z)\right\}
$$

or

$$
v(x, t)=\left(u_{0}^{*}+t H\right)^{*}(x) .
$$

The Hopf formula defines a convex function, which is a solution of (1) for a.e. $(x, t)$ in its domain. This domain will be all of $\mathbf{R}^{N}$, provided that the following property is fulfilled:

$$
\lim _{|p| \rightarrow+\infty} \frac{u_{0}^{*}(p)+t H(p)}{|p|}=+\infty, \quad \text { uniformly for } t \text { in }[0, T] .
$$

The following shows that $v$ is a viscosity solution of (1): This fact (under slightly less general assumptions) was proved in Bardi and Evans [1], but our method is simpler and more direct.

Proposition 1. Under assumption (8), if $u_{0}$ is convex and $u_{0}, H$ are continuous, the Hopf formula defines a viscosity solution of (1).

REMARK. Of course, if $u_{0} \in \mathrm{UC}\left(\mathbf{R}^{N}\right), u_{0}$ is in fact Lipschitz on $\mathbf{R}^{N}$, and, thus, $v$ given by (7), satisfies $D_{x} v \in L^{\infty}\left(\mathbf{R}^{N} \times\right] 0, T[)$. Then $v$ is the unique viscosity solution with this regularity in view of $[4,6$, and 2$]$.

Proposition 1 is a consequence of the following lemma, which ensures that the Hopf formula defines a semigroup.

LEMMA 1. For any functions $u_{0}, H$ from $\mathbf{R}^{N}$ to $\mathbf{R}$ and any positive numbers $t, s$ we have

$$
\left(\left(u_{0}^{*}+t H\right)^{* *}+s H\right)^{*}=\left(u_{0}^{*}+(t+s) H\right)^{*} \text {. }
$$

Proof of Lemma 1. Since for all $u, u^{* *} \leqslant u$, and since Fenchel's transformation is order-reversing, we have

$$
\left(u_{0}^{*}+t H\right)^{* *}+s H \leqslant u_{0}^{*}+(t+s) H
$$

and

$$
\left(\left(u_{0}^{*}+t H\right)^{* *}+s H\right)^{*} \geqslant\left(u_{0}^{*}+(t+s) H\right)^{*} .
$$

For the other inequality let us remark that

$$
\frac{s}{t+s} u_{0}^{*}+\frac{t}{t+s}\left(u_{0}^{*}+(t+s) H\right)^{* *} \leqslant u_{0}^{*}+t H .
$$

Since the left side is convex, we get

$$
\frac{s}{t+s} u_{0}^{*}+\frac{t}{t+s}\left(u_{0}^{*}+(t+s) H\right)^{* *} \leqslant\left(u_{0}^{*}+t H\right)^{* *} \text {. }
$$

Thus

$$
\left(u_{0}^{*}+(t+s) H\right)^{* *}-\left(u_{0}^{*}+t H\right)^{* *} \leqslant(s / t)\left[\left(u_{0}^{*}+t H\right)^{* *}-u_{0}^{*}\right] \leqslant s H .
$$


Consequently,

$$
\left(u_{0}^{*}+(t+s) H\right)^{* *} \leqslant\left(u_{0}^{*}+t H\right)^{* *}+s H .
$$

Taking conjugates, we obtain our second inequality.

Proof of Proposition 1. We already know that (7) defines a convex function satisfying (1) at each point of differentiability. In particular, it is a viscosity subsolution of (1) for if $v$ is superdifferentiable at $(x, t)$ then it is also differentiable and, thus,

$$
(\partial v / \partial t)(x, t)+H(D v(x, t))=0
$$

(this is in fact a very special case of arguments given in Lions [8]). Let us now prove that $v$ is also a viscosity supersolution. In other words, we have to show that, for any $\left(x_{0}, t_{0}\right)$ in $\mathbf{R}^{N} \times[0, T]$ and $(p, q)$ in the subdifferential of $v$ at $\left(x_{0}, t_{0}\right)$, we have

$$
q+H(p) \geqslant 0 \text {. }
$$

To prove this inequality, we adapt the arguments of Lions and Nisio [9]. By convexity and the definition of the subdifferential of $v$, we have

$$
\forall(x, t) \in \mathbf{R}^{N} \times[0, T], \quad v(x, t) \geqslant v\left(x_{0}, t_{0}\right)+\left(p, x-x_{0}\right)+q\left(t-t_{0}\right) .
$$

Let us denote by $\bar{S}_{H}(t)$ the semigroup defined by (8):

$$
\bar{S}_{H}(t) u_{0}=\left(u_{0}^{*}+t H\right)^{*} .
$$

By Lemma 1 we have for any $s$ in $\left[0, t_{0}\right]$,

$$
v\left(x_{0}, t_{0}\right)=\left[\bar{S}_{H}(s) v\left(\cdot, t_{0}-s\right)\right]\left(x_{0}\right) .
$$

Since $\bar{S}_{H}(s)$ is order preserving, we get, by (10),

$$
v\left(x_{0}, t_{0}\right) \geqslant\left(\bar{S}_{H}(s) \varphi\right)\left(x_{0}\right),
$$

where $\varphi(x)=v\left(x_{0}, t_{0}\right)+\left(p, x-x_{0}\right)-s q$. Thus

$$
v\left(x_{0}, t_{0}\right) \geqslant \sup _{z \in \mathbf{R}^{N}}\left\{\left(z, x_{0}\right)-t_{0} H(z)-\varphi^{*}(z)\right\} .
$$

But

$$
\varphi^{*}(z)= \begin{cases}s q-v\left(x_{0}, t_{0}\right)+p x_{0} & \text { if } z=p, \\ +\infty & \text { if } z \neq p .\end{cases}
$$

Consequently, $v\left(x_{0}, t_{0}\right) \geqslant-s q-t_{0} H(p)+v\left(x_{0}, t_{0}\right)$. Thus, for any $s$ in $\left[0, t_{0}\right]$ we get

$$
s q+t_{0} H(p) \geqslant 0 \text {, }
$$

which implies, in particular, that $q+H(p) \geqslant 0$.

REMARK. If we consider the natural extension of (1)

$$
\begin{array}{ll}
\partial u / \partial t+H(t, D u)=0 & \text { in } \left.\mathbf{R}^{N} \times\right] 0, T[, \\
u(x, 0)=u_{0}(x) & \text { in } \mathbf{R}^{N},
\end{array}
$$

then a natural extension of the Hopf formula is

$$
v(x, t)=\sup _{z \in \mathbf{R}^{N}}\left\{(x, z)-u_{0}^{*}(z)-\int_{0}^{t} H(s, z) d s\right\}
$$


or

$$
v(x, t)=\left(u_{0}^{*}+\int_{0}^{t} H(s, \cdot) d s\right)^{*}(x) .
$$

But $\left(6^{\prime}\right),\left(7^{\prime}\right)$ do not define a viscosity solution of (1); indeed, if this were the case, this formula would define an evolution operator and, by a density argument $\left(u_{0}^{*} \rightarrow 0\right)$, we would have

$$
\left\{\left(\int_{0}^{t} H(\lambda, \cdot) d \lambda\right)^{* *}+\int_{t}^{t+s} H(\lambda, \cdot) d \lambda\right\}^{*}=\left(\int_{0}^{t+s} H(\lambda, \cdot) d \lambda\right)^{*}
$$

for all $t, s \geqslant 0$, and this is, in general, false!

II. Commutation of the semigroups. The Hopf formula sheds some light on a new property of commutation of the semigroups:

Proposition 2. If $u_{0}, H_{1}, H_{2}$ are convex, continuous, and such that (8) holds for $H_{1}$ and $H_{2}$, then we have for all positive $t, s$,

$$
S_{H_{1}}(t) S_{H_{2}}(s) u_{0}=S_{H_{2}}(s) S_{H_{1}}(t) u_{0}=S_{t H_{1}+s H_{2}}(1) u_{0} .
$$

Proof. $S_{H_{1}}(t) S_{H_{2}}(s) u_{0}=\left[\left(u_{0}^{*}+s H_{2}\right)^{* *}+t H_{1}\right]^{*}$.

If $\mathrm{H}_{2}$ is convex this is equal to

$$
S_{t H_{1}+s H_{2}}(1) u_{0}=\left[u_{0}^{*}+t H_{1}+s H_{2}\right]^{*} \text {. }
$$

It is easy to find a counterexample to (11) if $H_{1}$ and $H_{2}$ are not convex. On the other hand, a reexamination of the Lax formula shows that the commutation property can also be proved for $u_{0}$ in $\operatorname{BUC}\left(\mathbf{R}^{N}\right)$ and convex continuous $H_{1}$ and $H_{2}$.

Proposition 3. If $u_{0} \in \mathrm{BUC}\left(\mathbf{R}^{N}\right)$ and $H_{1}, H_{2}$ are convex, then (11) holds for any positive $t, s$.

Proof. By the Oleinik-Lax formula we have

$$
\left(S_{H_{1}}(t) S_{I_{2}}(s) u_{0}\right)(x)=\operatorname{Inf}_{z \in \mathbf{R}^{N}} \operatorname{Inf}_{y \in \mathbf{R}^{N}}\left\{u_{0}(y)+\left(t H_{1}\right)^{*}(x-z)+\left(s H_{2}\right)^{*}(z-y)\right\} .
$$

But

$$
\operatorname{Inf}_{z \in \mathbf{R}^{N}}\left\{\left(t H_{1}\right)^{*}(x-z)+\left(s H_{2}\right)^{*}(z-y)\right\}=\left(t H_{1}+s H_{2}\right)^{*}(x-y) .
$$

Thus

$$
\left(S_{H_{1}}(t) S_{H_{2}}(s) u_{0}\right)(x)=\left(S_{t H_{1}+s H_{2}}(1) u_{0}\right)(x) .
$$

REMARK. The same commutation property obviously holds for small $t$, if $u_{0}$, $H_{1}, H_{2}$ are smooth; indeed by the method of characteristics (see, for example, [8]) we have the following: Let $v=S_{t H_{1}+s H_{2}}(1) u_{0}(x)$. Then for $t, s$ small there exists a unique $x_{1}$ in $\mathbf{R}^{N}$ such that

$$
\begin{gathered}
x_{1}+t H_{1}^{\prime}\left(D u_{0}\left(x_{1}\right)\right)+s H_{2}^{\prime}\left(D u_{0}\left(x_{1}\right)\right)=x, \quad D u_{0}\left(x_{1}\right)=D_{x} v(x, t, s), \\
v(x, t, s)=u_{0}\left(x_{1}\right)+t\left\{H_{1}^{\prime} \cdot D u_{0}\left(x_{1}\right)-H_{1}\right\}+s\left\{H_{2}^{\prime} \cdot D u_{0}\left(x_{1}\right)-H_{2}\right\} .
\end{gathered}
$$


Next if we set $x_{2}=x_{1}+t H_{1}^{\prime}\left(D u_{0}\left(x_{1}\right)\right)$, we have

$D u_{0}\left(x_{1}\right)=D_{x}\left(S_{H_{1}}(t) u_{0}\right)\left(x_{2}\right), S_{H_{1}}(t) u_{0}\left(x_{2}\right)=u_{0}\left(x_{1}\right)+t\left\{H_{1}^{\prime} \cdot D u_{0}\left(x_{1}\right)-H_{1}\right\}$, and observing that $x=x_{2}+s H_{2}^{\prime}\left(D_{x}\left(S_{H_{1}}(t) u_{0}\right)\left(x_{2}\right)\right)$, we conclude that

$$
v(x, t, s)=S_{H_{2}}(s) S_{H_{1}}(t) u_{0}(x) \text { for small } t, s \geqslant 0 \text {. }
$$

Still for small $t, s$, the same property would hold for general Hamiltonians $H_{1}(x, p)$, $H_{2}(x, p)$, provided we have

$$
\frac{\partial H_{1}}{\partial p} \cdot \frac{\partial H_{2}}{\partial x}-\frac{\partial H_{1}}{\partial x} \cdot \frac{\partial H_{2}}{\partial p}=0 \quad \text { on } \mathbf{R}^{N} \times \mathbf{R}^{N} ;
$$

i.e., $\left[H_{1}, H_{2}\right]=0\left(H_{1}, H_{2}\right.$ are in involution)!

III. Multitime equations. Problems arising in mathematical economics [10] lead to the following Cauchy problem for what we call (by analogy) the multitime Hamilton-Jacobi equation:

$$
\begin{array}{ll}
\partial u / \partial t+H_{1}(D u)=0 & \text { in } \mathbf{R}^{N} \times[0, T]^{2}, \\
\partial u / \partial s+H_{2}(D u)=0 & \text { in } \mathbf{R}^{N} \times[0, T]^{2}, \\
u(x, 0,0)=u_{0}(x) & \text { in } \mathbf{R}^{N} .
\end{array}
$$

Notice that (12) is apparently an overdetermined system of p.d.e.'s.

As a consequence of Propositions 2 and 3, we obtain explicit formulae giving weak solutions of (12).

Proposition 4. If $u_{0}$ is convex on $\mathbf{R}^{N}, u_{0}, H_{1}, H_{2}$ are continuous and if (8) holds for $H_{1}$ and $H_{2}$ then formula (13) defines a convex function $v$ on $\mathbf{R}^{N} \times[0, T]^{2}$, solving (12) a.e.

$$
v(x, t, s)=\left(u_{0}^{*}+t H_{1}+s H_{2}\right)^{*} .
$$

Proposition 5. If $u_{0} \in \mathrm{BUC}\left(\mathbf{R}^{N}\right), H_{1}, H_{2}$ are convex continuous, and if either $D u_{0} \in L^{\infty}$, or $H_{1}, H_{2}$ are coercive, then

$$
V=S_{H_{1}}(t) S_{H_{2}}(s) u_{0}=S_{H_{2}}(s) S_{H_{1}}(t) u_{0}=S_{t H_{1}+s H_{2}}(1) u_{0}
$$

is Lipschitz on $\mathbf{R}^{N} \times[\varepsilon, T]^{2}$ for any $\varepsilon>0$ and solves (12) a.e.

RemarKs. (i) In Proposition 4, for fixed $t>0, v$ is not, in general, a viscosity solution of $\partial u / \partial s+H_{2}(D u)=0$, although this is true in Proposition 5. However, in both cases $v=S_{t H_{1}+s H_{2}}(1) u_{0}$, and thus $v$ is a viscosity solution on each half-line connecting $(0,0)$ with $(t, s)$.

(ii) If $u_{0}, H_{1}, H_{2}$ are smooth (say $W^{2, \infty}\left(\mathbf{R}^{N}\right)$ ), then we may apply the method of characteristics (cf. Remark in §II), which yields that $v=S_{H_{1}}(t) S_{H_{2}}(s) u_{0}$ is a smooth solution of (12) provided $T$ is small $\left(T<T_{0}\left(u_{0}, H_{1}, H_{2}\right)\right)$.

(iii) Except for these special cases, we do not know if (12) admits global solutions for every $H_{1}, H_{2}, u_{0}$ : A good tentative solution could be $S_{t H_{1}+s H_{2}}(1) u_{0}$, but we are unable to decide if it solves (12) a.e.

(iv) Of course, Propositions 4-5 extend to an arbitrary number of times-that is, $m$ equations in $\mathbf{R}^{N} \times[0, T]^{m}$ involving $m$ different Hamiltonians for a single unknown function. 


\section{REFERENCES}

1. M. Bardi and L. C. Evans, On Hopf's formulas for solutions of Hamilton-Jacobi equations, preprint.

2. M. G. Crandall and P. L. Lions, Conditions d'unicité pour les solutions généralisées des équations de Hamilton-Jacobi du premier ordre, C. R. Acad. Sci. Paris 292 (1981), 183-186.

3. __ Viscosity solutions of Hamilton-Jacobi equations, Trans. Amer. Math. Soc. 277 (1983), 1-42.

4. Solutions de viscosité non bornées des équations de Hamilton-Jacobi du premier ordre, C. R. Acad. Sci. Paris (in preparation).

5. E. Hopf, Generalized solutions of nonlinear equations of first order, J. Math. Mech. 14 (1965), 951-973.

6. H. Ishii, Uniqueness of unbounded viscosity solutions of Hamilton-Jacobi equations, preprint.

7. P. D. Lax, Hyperbolic systems of conservation laws. II, Comm. Pure Appl. Math. 10 (1957), 537-566.

8. P. L. Lions, Generalized solutions of Hamilton-Jacobi equations, Pitman, London, 1982.

9. P. L. Lions and M. Nisio, A uniqueness result for the semigroup associated with the Hamilton Jacobi Bellman operation, Proc. Japan Acad. Math. Sci. 58 (1982), 273-276.

10. J. C. Rochet, The taxation principle and multi-time Hamilton-Jacobi equations, preprint, Univ. Paris IX.

Ceremade, Paris IX University, Place de Lattre de Tassigny, 75775 Paris Cedex 16, France 